\title{
Resource Allocation for Vertical Sectorization in LTE-Advanced Systems
}

\author{
Lei Song, Mugen Peng, and Yan Li \\ Wireless Signal Processing and Network Lab, Key Laboratory of Universal Wireless Communications (Ministry of Education), \\ Beijing University of Posts and Telecommunications, Beijing 100876, China
}

Correspondence should be addressed to Mugen Peng; pmg@bupt.edu.cn

Received 28 October 2013; Accepted 17 November 2013

Academic Editor: Zhaobiao Lv

Copyright (C) 2013 Lei Song et al. This is an open access article distributed under the Creative Commons Attribution License, which permits unrestricted use, distribution, and reproduction in any medium, provided the original work is properly cited.

Massive multiple input multiple output (MIMO) technology has been discussed widely in the past few years. Three-dimensional MIMO (3D MIMO) can be seen as a promising technique to realize massive MIMO to enhance the performance of LTE-Advanced systems. Vertical sectorization can be introduced by means of adjusting the downtilt of transmitting antennas. Thus, the radiowave from a base station (BS) to a group of user equipments (UE) can be divided into two beams which point at two different areas within a cell. Intrasector interference is inevitable since the resources are overlapped. In this paper, the influence of intrasector interference is analyzed and an enhanced resource allocation scheme for vertical sectorization is proposed as a method of interference cancellation. Compared with the conventional 2D MIMO scenarios, cell average throughput of the whole system can be improved by vertical sectorization. System level simulation is performed to evaluate the performance of the proposed scheme. In addition, the impacts of downtilt parameters and intersite distance (ISD) on spectral efficiency and cell coverage are presented.

\section{Introduction}

Long Term Evolution (LTE), proposed by the Third Generation Partnership Project (3GPP), is aimed at achieving enhanced performance in terms of cell throughput and system overhead. As is generally known, LTE-Advanced is addressed to achieve higher data rates and better UEs' Quality of Service (QoS) than LTE $[1,2]$. Based on Orthogonal Frequency Division Multiple Access (OFDMA), capacity of wireless systems can be significantly increased by using multiantenna methods. As one of the key techniques in LTEAdvanced systems, massive MIMO has been widely discussed currently and 3D MIMO can be seen as an effective method to approach large-scale MIMO [3-6]. Compared with the conventional 2D antenna, 3D antenna is comprised of many element units. Each element is deployed at different heights in an individual 3D antenna. Thus, difference of elements in vertical domain should not be neglected.

Benefiting from Active Antenna Systems (AAS), many active antenna technologies such as 3D beamforming and vertical sectorization could be easily implemented. Vertical sectorization can be realized by multiple antenna elements, by which two separate beams with their distinct antenna parameters are arranged to serve vertically split inner and outer cells. In our work, performance with various electrical tilt angles have been simulated to observe the optimization space of the overlap between two vertical sectors and the gain in terms of cell capacity.

One of the major advantages of introducing vertical sectorization into LTE-Advanced systems is to improve cell capacity. In [7], capacity gain brought by electrical downtilt in standard 3-sector BS (Base Station) configuration could be up to $48.4 \%$ for $1500 \mathrm{~m}$ intersite distance for 3G WCDMA systems. In [8], 45.7\% capacity improvement was observed for 3-sector BS with $1732 \mathrm{~m}$ intersite distance by optimizing antenna parameters for LTE systems. In [9], performance of vertical sectorization with Near Electrical Tilt (NET) and Remote Electrical Tilt (RET) is evaluated for different network deployment scenarios by means of 3D system level simulation.

By applying the separate antenna elements into transmitter, each BS can dominate twice as many frequency resources 


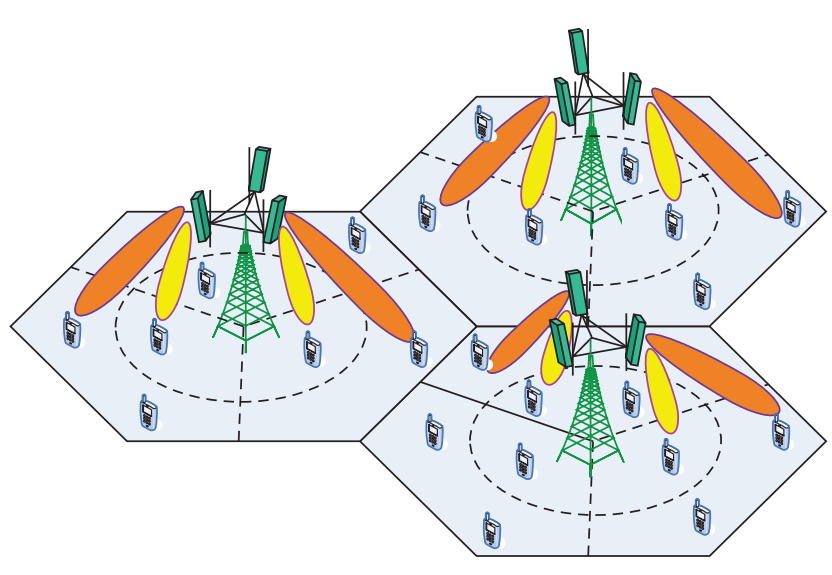

Figure 1: vertical sectorization and $3 \times 2$ sector layout.

as before. The number of physical resource blocks (PRBs) is doubled for vertical sectorization networks. Severe intracell interference will occur to reduce the signal to interference and noise ratio (SINR) of UEs and degrade performance of the whole networks when the resources are scheduled to different groups of UEs at the same time. In this paper, an enhanced resource allocation scheme based on coordinated communication is proposed. Compared with the traditional frequency reuse scheme, the proposed scheme is able to eliminate severe intracell interference and hence provide better SINR of UE.

The rest of this paper is organized as follows. Section 2 describes the system model of the vertical sectorization in LTE-Advanced systems and the deployment details. Section 3 proposes an enhanced scheduling scheme for vertical sectorization, and performance of the proposed scheme with different scenario parameters is simulated and analyzed in Section 4. The conclusion of this paper is given in Section 5.

\section{System Model}

Since a cell has 3 sectors and each sector only has one BS in the regular systems, the LTE-Advanced systems without vertical sectorization are referred to as $3 \times 1$ networks. The LTEAdvanced systems with vertical sectorization are referred to as $3 \times 2$ networks since each sector is partitioned into 2 subsectors, as is shown in Figure 1.

It is assumed that the total transmission power of a sector of $3 \times 2$ networks remains the same as $3 \times 1$ networks, and the power is equally split between two subsectors. The signal lobe radiated by the antenna is split into two lobes with different downtilts. The first lobe serving the UEs near BS is generated by the bottom row of antenna elements by using a higher downtilt (i.e., NET), while the second one serving the UEs close to cell border is generated by the top row of antenna elements using a lower downtilt (i.e., RET). The same system bandwidth is used for both subsectors. The amount of resources is doubled, but the power per physical resource block (PRB) is halved.

Supposing that there are total $M$ hexagonal cells with $K$ active UEs served within the coverage of each sector. The total number of BSs in the system is $3 M$. Each BS which corresponds to one sector is equipped with $N_{t}$ transmitting antennas, while each UE has $N_{r}$ receiving antennas. For each sector divided into inner sector and outer sector, the bottom and the top rows of antenna elements can be seen as inner sector and outer sector antenna elements, respectively. There are two groups of UEs in one sector: inner sector UEs and outer sector UEs, which means that UEs are located near the serving BS and far away from the serving BS, respectively. Thus, taking inner sector UE, for example, the received signal at the $k$ th UE served by BS $m$ is as follows:

$$
\begin{aligned}
\mathbf{y}_{m \text {-inner }}^{(k)}= & \underbrace{\mathbf{H}_{m \text {-inner }}^{(k)} \mathbf{W}_{m \text {-inner }}^{(k)} \mathbf{s}_{m \text {-inner }}^{(k)}}_{\text {desired signal }}+\underbrace{\mathbf{H}_{m \text {-outer }}^{(k)} \mathbf{W}_{m \text {-outer }}^{(k)} \mathbf{s}_{m \text {-outer }}^{\left(k^{\prime}\right)}}_{\text {intrasector interference }} \\
& +\underbrace{\sum_{n \neq m}^{3 M} \sum_{w=1}^{K} \mathbf{H}_{n \text {-inner }}^{(k)} \mathbf{W}_{n \text {-inner }}^{(k)} \mathbf{s}_{n \text {-inner }}^{(w)}+\sum_{n \neq m}^{3 M} \sum_{w=1}^{K} \mathbf{H}_{n \text {-outer }}^{(k)} \mathbf{W}_{n \text {-outer }}^{(k)} \mathbf{s}_{n \text {-outer }}^{(w)}}_{\text {inter-sector interference }}+\mathbf{n}_{m}^{(k)},
\end{aligned}
$$

where $\mathbf{s}_{m \text {-inner }}^{(k)}$ is the $l \times 1$ transmitted vector for inner sector UE $k$ served by BS $m$ and $l$ is the number of data streams. $\mathbf{s}_{m \text {-inner }}^{(k)}=\left[\sqrt{p_{k}} s_{m, 1}^{(k)}, \sqrt{p_{k}} s_{m, 2}^{(k)}, \ldots, \sqrt{p_{k}} s_{m, l}^{(k)}\right]^{T}$, and $p_{k}$ is the transmit power of each data stream at UE $k$. It is assumed that each transmitting antenna has $N_{v}$ elements in vertical dimension and the receiving antennas are still conventional 2D antennas. Thus, $\mathbf{H}_{m \text {-inner }}^{(k)}$ is the $\left(N_{r} \times N_{t}\right)$ channel matrix from the inner sector antenna elements in BS $m$ to UE $k$ and $\mathbf{W}_{m \text {-inner }}^{(k)}$ is the $\left(N_{t} \times l\right)$ precoding matrix. $\mathbf{n}_{m}^{(k)}$ is the additive white Gaussian noise with zero mean and variance $\mathbb{E}\left(\mathbf{n}_{m}^{(k)} \mathbf{n}_{m}^{(k) H}\right)=\sigma^{2}$.

\section{Proposed Scheme for Vertical Sectorization}

3.1. Intracell Interference Brought by Vertical Sectorization. For $3 \times 1$ networks, detailed analysis of detected SINR for the received signal is in [10]. Due to the introduction of vertical sectorization as shown in Figure 2, the received power of UE is halved and the source of interference is much more complex than before. The expression of the SINR can be depicted as follows: 


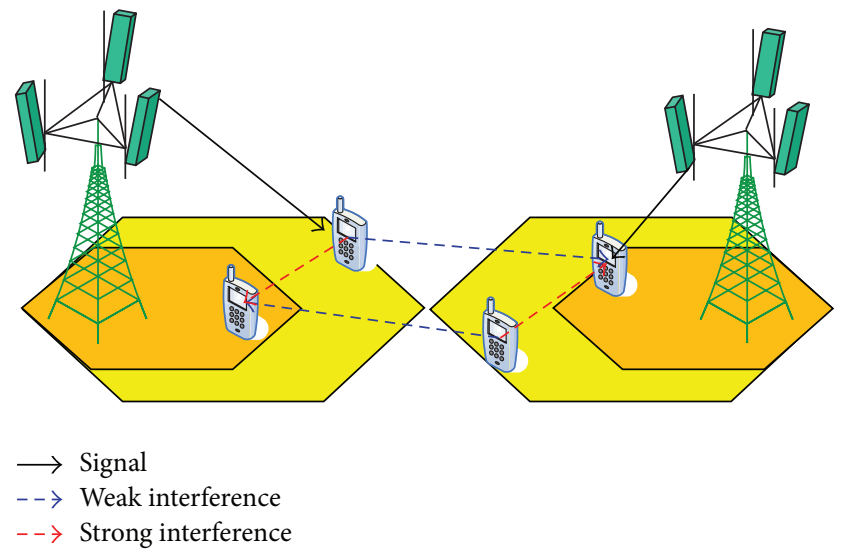

FIGURE 2: Limitation of vertical sectorization.

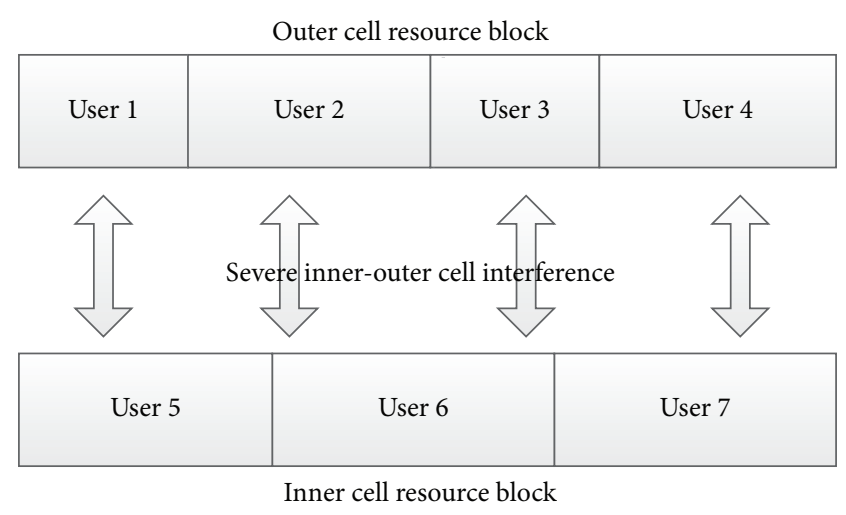

FIGURE 3: Intrasector interference brought by vertical sectorization.

$$
\operatorname{SINR}^{k}=\frac{P_{L}^{k} \mathbf{W}^{H} \mathbf{H}_{m \text {-inner }}^{k}\left(\mathbf{H}_{m \text {-inner }}^{k}\right)^{H} \mathbf{W}}{\underbrace{\sum_{n=0, n \neq m}^{3 M} P_{L}^{k} \mathbf{W}^{H} \mathbf{H}_{n}^{k}\left(\mathbf{H}_{n}^{k}\right)^{H} \mathbf{W}}_{\text {inter-sector interference }}+\underbrace{P_{L}^{k^{\prime}} \mathbf{W}^{H} \mathbf{H}_{m \text {-outer }}^{k}\left(\mathbf{H}_{m-\text { outer }}^{k}\right)^{H} \mathbf{W}}_{\text {intrasector interference }}+P_{\text {noise }}},
$$

where $P_{L}^{k}=P_{t x}^{k} P_{\text {loss }}^{k} / 2, P_{\text {noise }}=\sigma^{2} \mathbf{W}^{H} \mathbf{W}$.

3.2. Previous Work on Resource Allocation. For a sector in vertical sectorization networks, the inner sector and outer sector independently schedule radio resources for the UEs in their respective dominance areas. Figure 3 illustrates an example of a possible scheduling condition. The signals become mutual interference to most UEs and such scheduling confliction could happen all the time. Furthermore, the path losses experienced by UEs belonging to inner sector or outer sector are basically identical because the two beams are radiated from the same active antenna. Therefore, the received signal and interference are mainly determined by the vertical antenna gains. Thus, UEs in the area where two vertical antenna patterns overlap (defined as sector edge UEs in this paper) will suffer severe Intrasector interference.

Fortunately, the same active antenna serving inner sector and outer sector gives us the opportunity to avoid Intrasector interference through cooperative scheduling. The previous scheduling scheme [11] is shown in Figure 4. Instead of scheduling resources independently by the inner sector and outer sector, the radio resources are scheduled orthogonally to UEs in the same manner of that in the $3 \times 1$ networks. In this case, the SINR of UEs would be improved largely.

3.3. Proposed Resource Allocation Scheme. As is analyzed in the previous subsection, without proper interference cancellation mechanism, the utilization of vertical sectorization would worsen the performance of sector-edge UEs. In order to provide better service for these UEs, an enhanced resource allocation scheme is presented in Figure 5. UE A and UE $B$ are both sector-edge UEs as mentioned above. Based on coordinated communication, the same part of resources from the two subsectors can be allocated to UE A. Another similar part of resources from the two subsectors orthogonal to those allocated to UE A can be allocated to UE B. In this case, for each UE, not only the Intrasector interference is eliminated, but the power of signal is also doubled. The mutual interference between sector-edge UEs can be avoided by frequency orthogonality. The specific methods to achieve the scheme are as follows.

Step 1. Divide the UEs accessed in vertical sectorization networks into three sets: sector-edge UE set, inner sector UE set, and outer sector UE set. Reference Signal Received Power (RSRP) principle is utilized to decide whether the UE belongs to sector-edge UE set. If the difference of RSRP between inner sector and outer sector is less than a settled threshold, the UE can be recognized as a sector-edge UE. The judging criteria can be expressed as follows:

$$
\begin{gathered}
\mid P_{\mathrm{BS}} \times G_{\text {loss }} \times G_{\text {shadow }} \times G_{\text {inner-antenna }}-P_{\mathrm{BS}} \\
\times G_{\text {loss }} \times G_{\text {shadow }} \times G_{\text {outer-antenna }} \mid<\text { Thr. }
\end{gathered}
$$

Step 2. Divide the whole resources in each sector into two sets, $G$ and $F$, where $G \cap F=\emptyset$. Resources in set $G$ are used for serving sector-edge UEs, while resources in set $F$ are used for inner sector UEs and outer sector UEs.

Step 3. For a sector, judge whether sector-edge UEs exist in the current scheduling period. If the number of sector-edge UEs $N_{\text {sector-edgeUE }}>0$, both inner sector and outer sector assign the PRBs in set $G$ to sector-edge UEs. After that, the resources in $F$ are allocated to other UEs. Otherwise, for no sector-edge UE case, PRBs in $G$ and $F$ are allocated to all UEs in the sector without any restriction. 


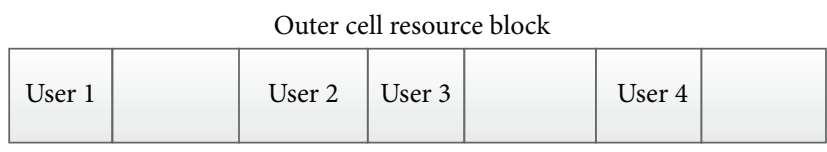

No inner-outer cell interference

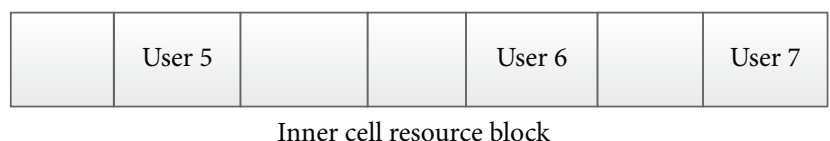

Figure 4: Previous scheduling scheme for vertical sectorization.

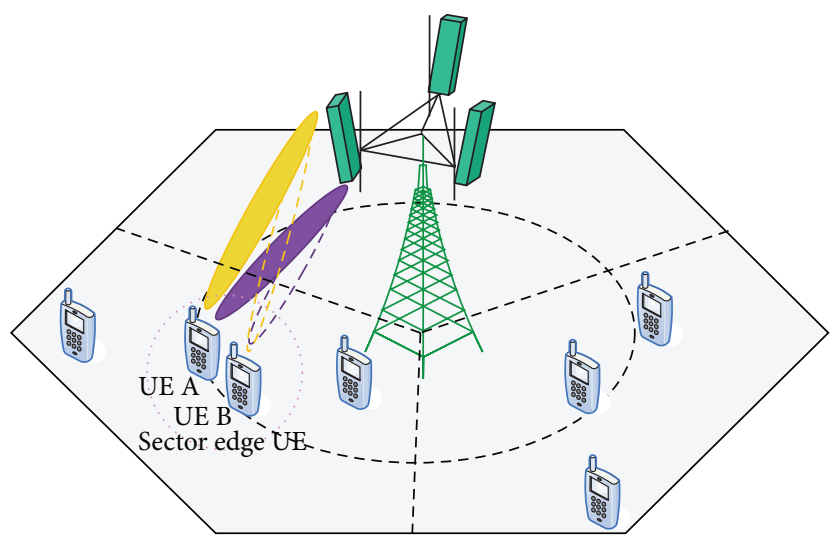

FIgURE 5: Proposed resource allocation scheme.

Step 4. Apply beamforming algorithm as a manner of joint precoding for sector-edge UEs. Take sector-edge UE $i$; for example, $\mathbf{h}_{\text {inner }}^{i}$ and $\mathbf{h}_{\text {outer }}^{i}$ are the channels generated by inner sector and outer sector antenna elements, respectively. The equivalent channel from sector $m$ to UE $i$ is defined as $\mathbf{H}_{\text {eff }}$, where $\mathbf{H}_{\text {eff }}=\left[\mathbf{h}_{\text {inner }}^{i} \mathbf{h}_{\text {outer }}^{i}\right] . \mathbf{V}_{i, m} \sim$ $\operatorname{SVD}\left(\mathbf{H}_{\mathrm{eff}}\right), \mathbf{V}_{i, m}$ is the $\mathrm{V}$ matrix from SVD decomposition of $\mathbf{H}_{\text {eff }}$. The beamforming vector $\mathbf{v}_{i, m}$ is the first column of matrix $\mathbf{V}_{i, m}$. The received signal can be derived:

$$
\mathbf{y}_{m}^{(i)}=\underbrace{\mathbf{H}_{e f f}^{(i)} \mathbf{v}_{i, m} \mathbf{s}_{m}^{(i)}}_{\text {desired signal }}+\underbrace{\sum_{n \neq m}^{3 M} \sum_{w=1}^{K} \mathbf{H}_{m \text {-inner }}^{(i)} \mathbf{W}_{m \text {-inner }}^{(i)} \mathbf{s}_{m}^{(w)}+\sum_{n \neq m}^{3 M} \sum_{w=1}^{K} \mathbf{H}_{m \text {-outer }}^{(i)} \mathbf{W}_{m \text {-outer }}^{(i)} \mathbf{s}_{m}^{(w)}}_{\text {inter-sector interference }}+\mathbf{n}_{m}^{(i)} .
$$

Thus, the SINR of sector-edge UE $i$ can be expressed as follows:

$$
\operatorname{SINR}^{k}=\underbrace{\frac{P_{L}^{k} \mathbf{W}^{H} \mathbf{H}_{m \text {-inner }}^{k}\left(\mathbf{H}_{m \text {-inner }}^{k}\right)^{H} \mathbf{W}+P_{L}^{k} \mathbf{W}^{H} \mathbf{H}_{m \text {-outer }}^{k}\left(\mathbf{H}_{m \text {-outer }}^{k}\right)^{H} \mathbf{W}}{\sum_{n=0, n \neq m}^{3 M} P_{L} \mathbf{W}^{H} \mathbf{H}_{n \text {-inner }}^{k}\left(\mathbf{H}_{n \text {-inner }}^{k}\right)^{H} \mathbf{W}+\sum_{n=0, n \neq m}^{3 M} P_{L} \mathbf{W}^{H} \mathbf{H}_{n \text {-outer }}^{k}\left(\mathbf{H}_{n \text {-outer }}^{k}\right)^{H} \mathbf{W}}+P_{\text {noise }}}_{\text {inter-sector interference }} .
$$

\section{Simulation Results}

In this section, system level performance of the proposed resource allocation scheme is evaluated in terms of cell average spectral efficiency and SINR of the UEs. Detailed simulation parameters are listed in Table 1.
Figure $6(a)$ is the $2 \times 2$ cell average spectral efficiency of different downtilt configurations with sector radius of $288 \mathrm{~m}$. For these downtilt configurations, the performance of $3 \mathrm{D}$ vertical sectorization has $16 \%, 27 \%, 15 \%$, and $26 \%$ enhancement compared to 2D MIMO networks, respectively. Although system capacity gain is decreased due to the orthogonality 


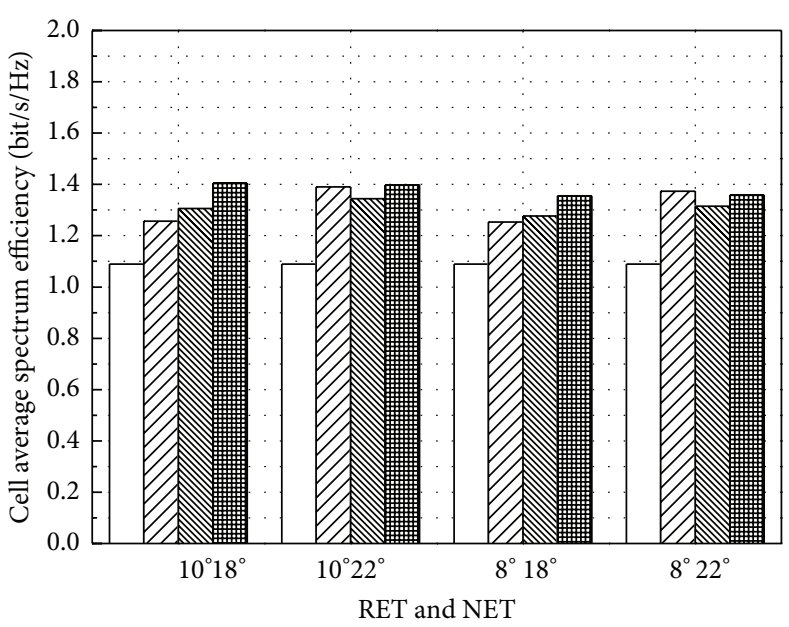

(a)

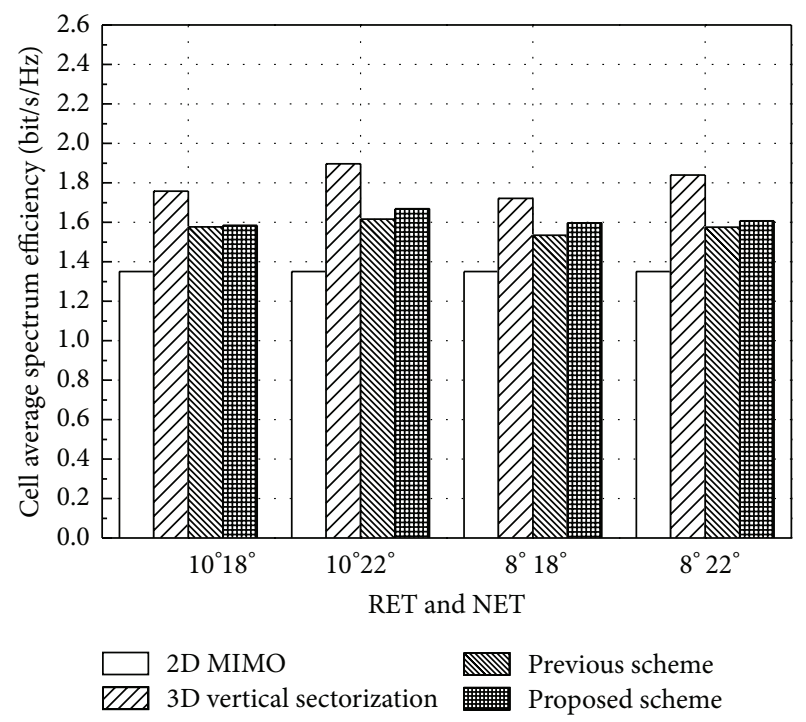

(c)

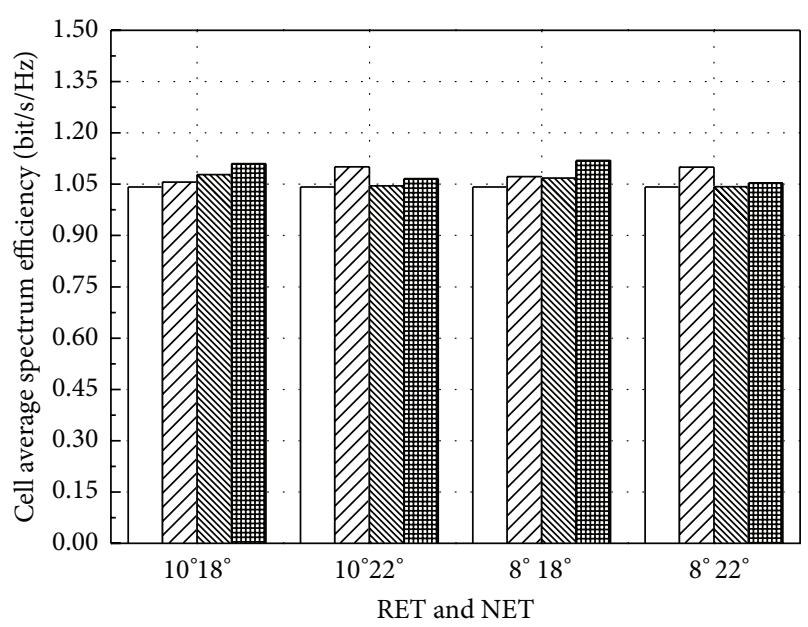

(b)

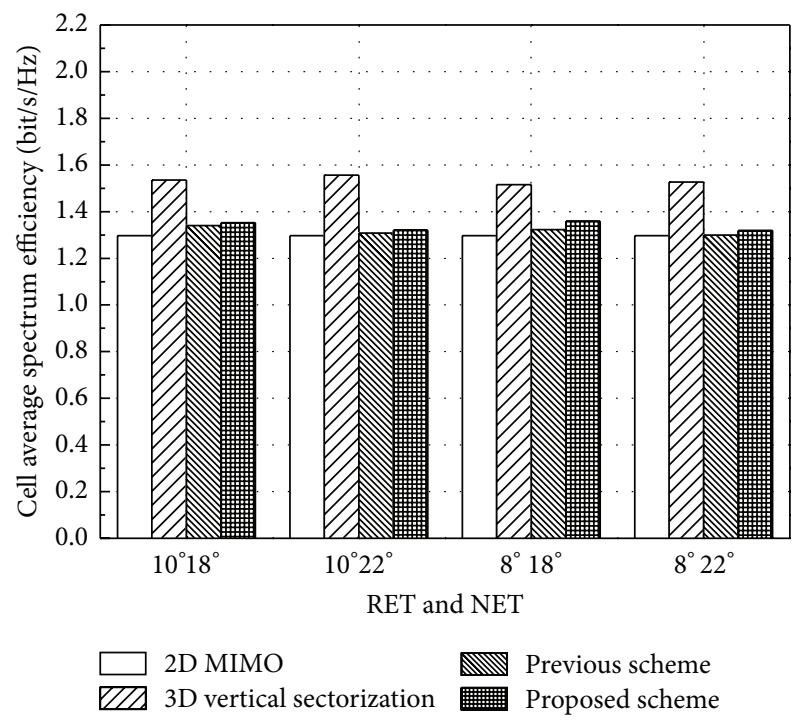

(d)

Figure 6: System level results of 2D MIMO, 3D vertical sectorization, previous scheme, and proposed scheme: (a) $2 \times 2$ cell average spectral efficiency with sector radius of $288 \mathrm{~m}$; (b) $2 \times 2$ cell average spectral efficiency with sector radius of $288 \mathrm{~m}$; (c) $4 \times 2$ cell average spectral efficiency with sector radius of $500 \mathrm{~m}$; (d) $4 \times 2$ cell average spectral efficiency with sector radius of $500 \mathrm{~m}$.

of resources, there is still a substantial improvement in the performance of throughput. Since coordinated transmission is applied into our proposed scheme, UEs located at the sector edge area are allocated twice as many resources as before. As can be seen in the figure, the proposed scheme is able to achieve an obvious gain in throughput compared to the previous resource allocation scheme. About 29\%, 28\%, $24 \%$, and $25 \%$ of the gain compared to $2 \mathrm{D}$ MIMO can be achieved by the enhanced scheme with different RET and NET configurations.

The cell average spectral efficiency of $2 \times 2500 \mathrm{~m}$ scenario is shown in Figure 6(b). Since the radius of sector increases, the distribution of UEs within a sector changes a lot and the elevation angles of UEs in the area decrease. The same RET and NET configuration cannot cover as many UEs as in $288 \mathrm{~m}$ scenario. Although the performance of coverage is not so good as Figure 6(a), the proposed scheme still achieves a performance gain compared to the previous scheme and $2 \mathrm{D}$ MIMO networks. Cell average spectral efficiency has utmost $8 \%$ enhancement compared to $2 \mathrm{D}$ MIMO networks.

The cell average spectral efficiency of $4 \times 2288 \mathrm{~m}$ scenario is shown in Figure 6(c). For each downtilt configuration, 3D vertical sectorization can achieve more than $30 \%$ enhancement compared with 2D MIMO networks. Since the resources of inner sector and outer sector are allocated to UEs in an orthogonal manner, the throughput of cell average 


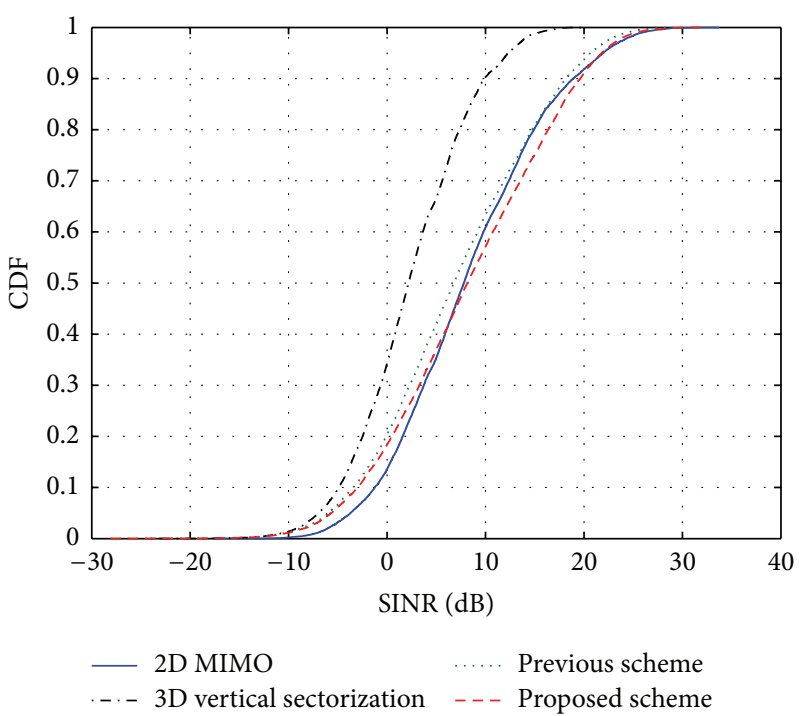

(a)

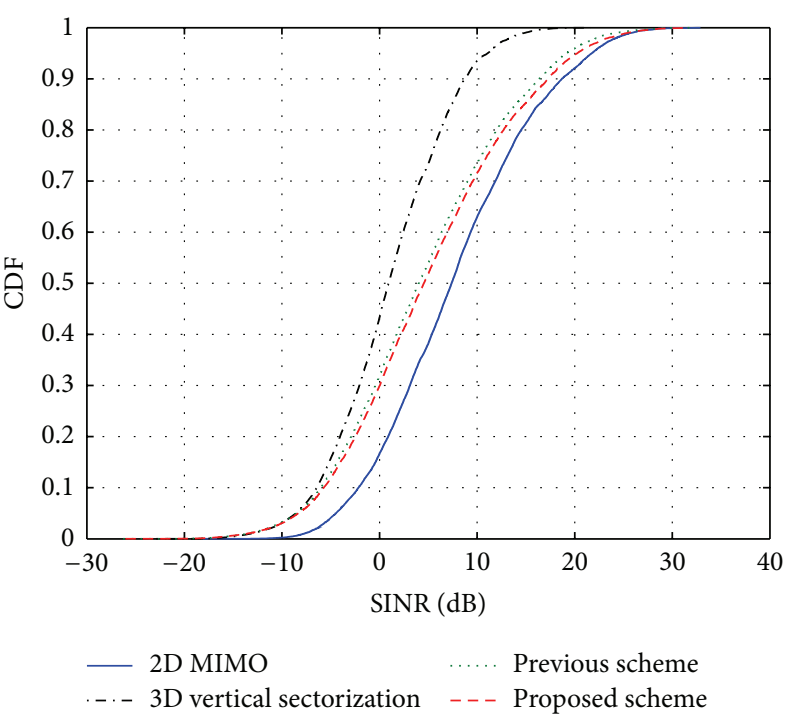

(b)

Figure 7: System level results of UEs' receive SINR CDF for 2D MIMO, 3D vertical sectorization, previous scheme, and proposed scheme: (a) $2 \times 2288 \mathrm{~m}$ scenario; (b) $2 \times 2500 \mathrm{~m}$ scenario.

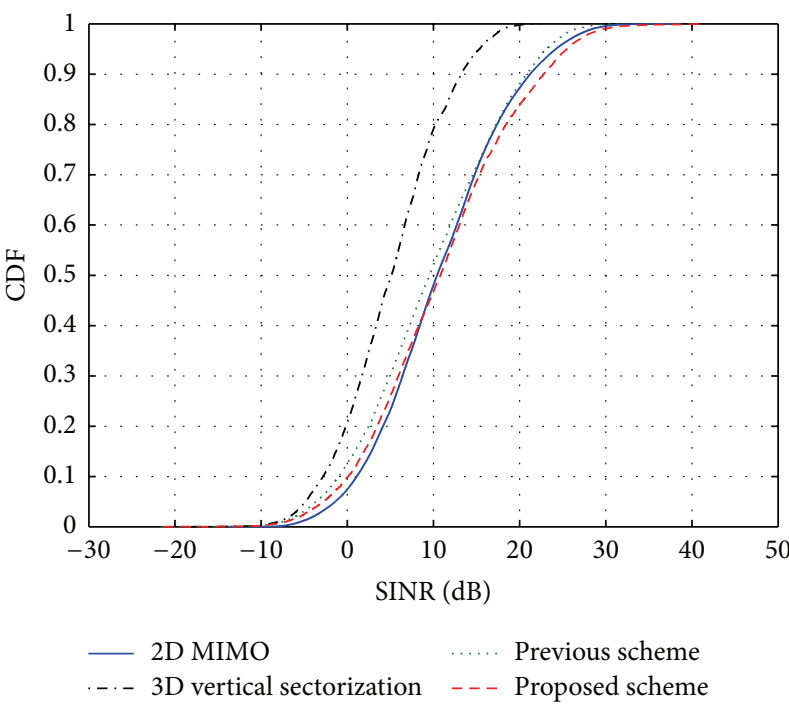

(a)

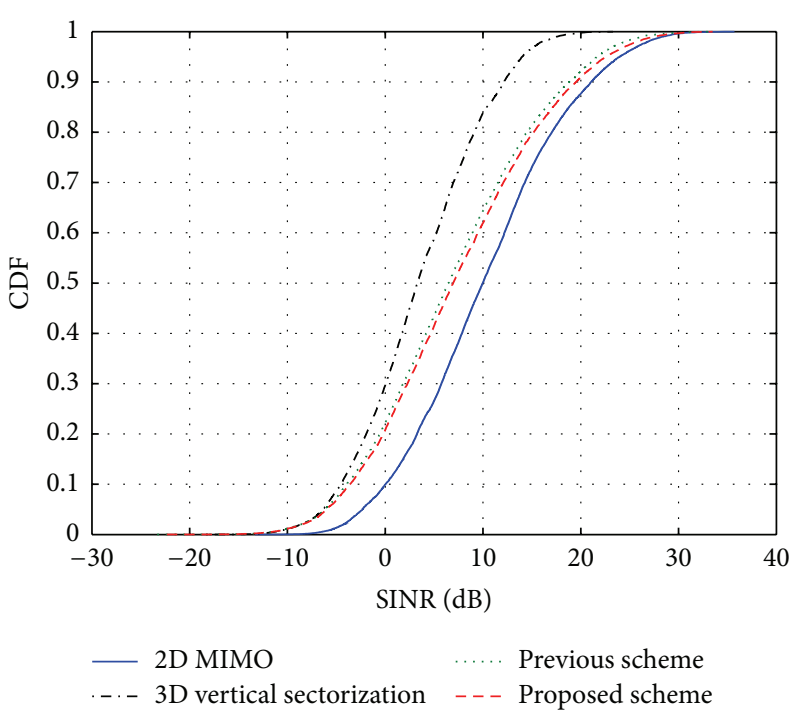

(b)

FIGURE 8: System level results of UEs' receive SINR CDF for 2D MIMO, 3D vertical sectorization, previous scheme, and proposed scheme: (a) $4 \times 2288 \mathrm{~m}$ scenario; (b) $4 \times 2500 \mathrm{~m}$ scenario.

UEs decreases. By applying the enhanced resource allocation, the proposed scheme outperforms the previous scheme in all the scenarios. It can be seen that as the increasing of sector radius, there are not too many sector edge UEs existing, so the throughput gain brought by the proposed scheme is not so obvious in the $500 \mathrm{~m}$ scenario as shown in Figure 6(d).
Figure 7 shows the UEs' receiving SINR CDF of $2 \mathrm{D}$ MIMO, 3D vertical sectorization, the previous scheme, and the proposed scheme. For $2 \times 2288 \mathrm{~m}$ scenario and $4 \times 2288 \mathrm{~m}$ scenario, performance of $3 \mathrm{D}$ vertical sectorization descends approximately $7 \mathrm{~dB}$ compared with that of $2 \mathrm{D}$ MIMO due to the introduction of sector interference. The average SINR 
TABLE 1: Simulation configuration parameters.

\begin{tabular}{lc}
\hline Parameters & Assumptions \\
\hline Cell type & Macrocell \\
Cellular layout & 19 cells, each with 3 sectors \\
Sector radius & $288 \mathrm{~m}, 500 \mathrm{~m}$ \\
Bandwidth & $5 \mathrm{M}$ \\
Channel model & WINNER II \\
Antenna number & Transmitter: $2 \mathrm{Tx}, 4 \mathrm{Tx}$ \\
Antenna configuration & Receiver: $2 \mathrm{Rx}$ \\
& Copolarized linear array \\
Antenna spacing & Transmitter: $4.0 \lambda$, \\
Number of elevation elements & Receiver: $0.5 \lambda$ \\
Regular antenna gain & 2 \\
BS max TX power & $14 \mathrm{dBi}$ \\
BS height & $46 \mathrm{dBm}$ \\
UE height & $32 \mathrm{~m}$ \\
UE numbers & $1.5 \mathrm{~m}$ \\
Scheduler & 10 UEs per sector \\
Receiver & Round-robin \\
Traffic model & MMSE \\
\hline
\end{tabular}

of the previous scheme outperforms that of $3 \mathrm{D}$ vertical sectorization by $5 \mathrm{~dB}$. Since sector edge UEs in the proposed scheme can be served much better than before, the average SINR of the proposed scheme has $1 \mathrm{~dB}$ gain over that of the previous scheme.

When the sector radius is increased, UEs are located farther away to each other and sector edge UEs' impacts on system performance are reduced largely. As is depicted in Figure 8, the UEs' average SINR of the two resource allocation schemes is less than that of 2D MIMO. In spite of this, the SINR of UEs in the proposed scheme and the previous scheme still outperform that of $3 \mathrm{D}$ vertical sectorization.

\section{Conclusion}

In this paper, modeling of active antenna and structure of vertical sectorization have been introduced. Compared with 2D MIMO scheme, vertical sectorization can enhance the cell throughput significantly but will suffer serious Intrasector interference. To solve this problem, the interference suffered by UEs is analyzed and an enhanced resource allocation scheme for vertical sectorization in LTE-Advanced systems is proposed. System level simulation has been performed to evaluate the performance of the proposed scheme. Simulation results can prove that it is an effective method to eliminate intracell interference and increase system capacity. Beyond that, simulation results for vertical sectorization with different downtilt parameters and sector radius parameters are presented, which indicate that the proposed scheme can improve the SINR of sector-edge UEs and maintain the cell average throughput gain.

\section{Acknowledgments}

This work was supported in part by the State Major Science and Technology Special Projects (Grant no. 2012ZX03001028004, 2013ZX03001001, and 2012ZX03001031), the National Natural Science Foundation of China (Grant no. 61222103, no. 61072058), the Beijing Natural Science Foundation (Grant no. 4131003), and the Program for New Century Excellent Talents in University (NCET-10-0262).

\section{References}

[1] M. Peng and W. Wang, "Technologies and standards for TDSCDMA evolutions to IMT-advanced," IEEE Communications Magazine, vol. 47, no. 12, pp. 50-58, 2009.

[2] M. Peng, D. Liang, Y. Wei, J. Li, and H. Chen, "Self-configuration and self-optimization in LTE-advanced heterogeneous networks," IEEE Communications Magazine, vol. 51, no. 5, pp. 36-45, 2013.

[3] F. Rusek, D. Persson, B. K. Lau et al., "Scaling up MIMO: opportunities and challenges with very large arrays," IEEE Signal Processing Magazine, vol. 30, no. 1, pp. 40-60, 2013.

[4] H. Huh, G. Caire, H. C. Papadopoulos, and S. A. Ramprashad, "Achieving "massive MIMO" spectral efficiency with a not-solarge number of antennas," IEEE Transactions on Wireless Communications, vol. 11, no. 9, pp. 3226-3239, 2012.

[5] M. Peng, X. Zhang, W. Wang, and H.-H. Chen, "Performance of dual-polarized MIMO for TD-HSPA evolution systems," IEEE Systems Journal, vol. 5, no. 3, pp. 406-416, 2011.

[6] T. Zhou, M. Peng, W. Wang, and H. Chen, "Low-complexity coordinated beamforming for downlink multi-cell SDMA/ OFDM System," IEEE Transactions on Vehicular Technology, vol. 62, no. 1, pp. 247-255, 2013.

[7] J. Niemelä, T. Isotalo, and J. Lempiäinen, "Optimum antenna downtilt angles for macrocellular WCDMA network," EURASIP Journal on Wireless Communications and Networking, vol. 2005, Article ID 610942, 2005.

[8] O. N. C. Yilmaz, S. Hämäläinen, and J. Hämäläinen, “Analysis of antenna parameter optimization space for 3GPP LTE," in Proceedings of the 70th Vehicular Technology Conference Fall (VTC '09-Fall), pp. 1-5, Anchorage, Alaska, USA, September 2009.

[9] O. N. C. Yilmaz, S. Hämäläinen, and J. Hämäläinen, "System level analysis of vertical sectorization for 3GPP LTE," in Proceedings of the 6th International Symposium on Wireless Communication Systems (ISWCS '09), pp. 453-457, Tuscany, Italy, September 2009.

[10] F. Shu, L. Lihua, C. Qimei, and Z. Ping, "Non-unitary codebook based precoding scheme for multi-user MIMO with limited feedback," in Proceedings of the IEEE Wireless Communications and Networking Conference (WCNC '08), pp. 678-682, Las Vegas, Nev, USA, April 2008.

[11] M. Peng, W. Wang, and H.-H. Chen, "Multiuser pairing-up schemes under power constraints for uplink virtual MIMO networks," Mobile Networks and Applications, vol. 15, no. 2, pp. 298-309, 2010. 

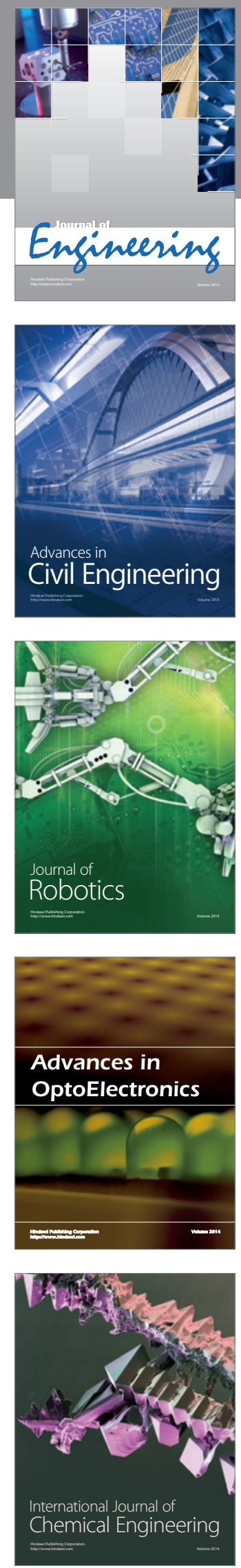

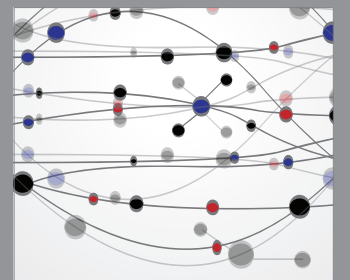

The Scientific World Journal
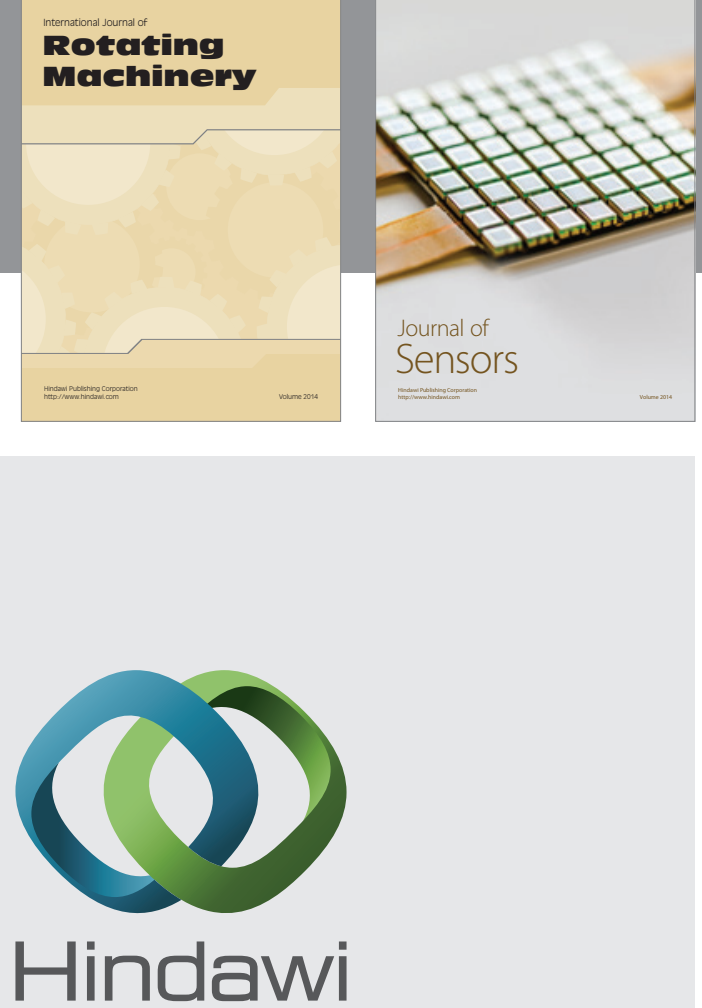

Submit your manuscripts at http://www.hindawi.com
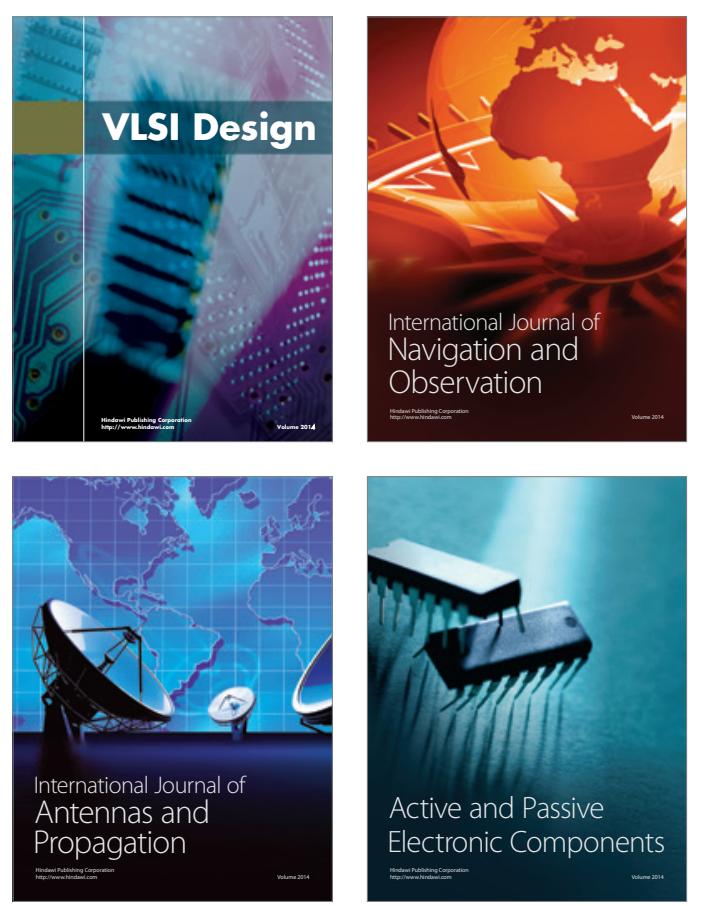
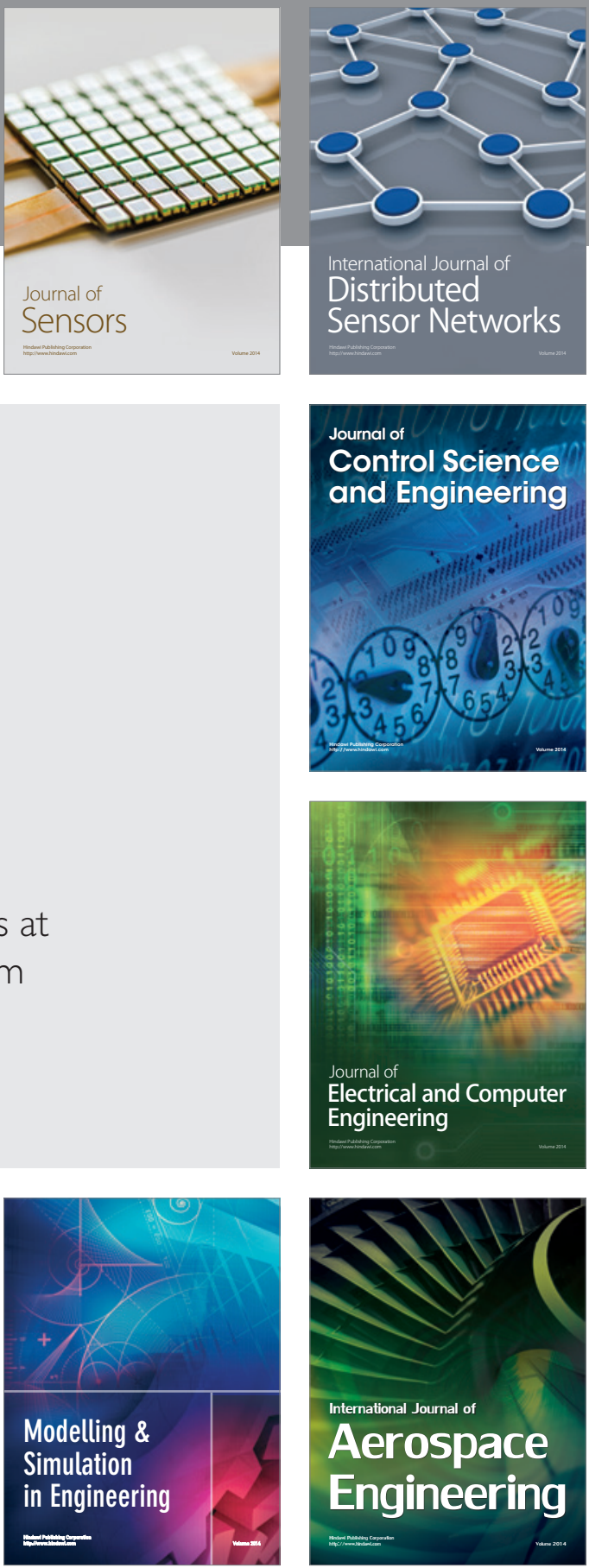

Journal of

Control Science

and Engineering
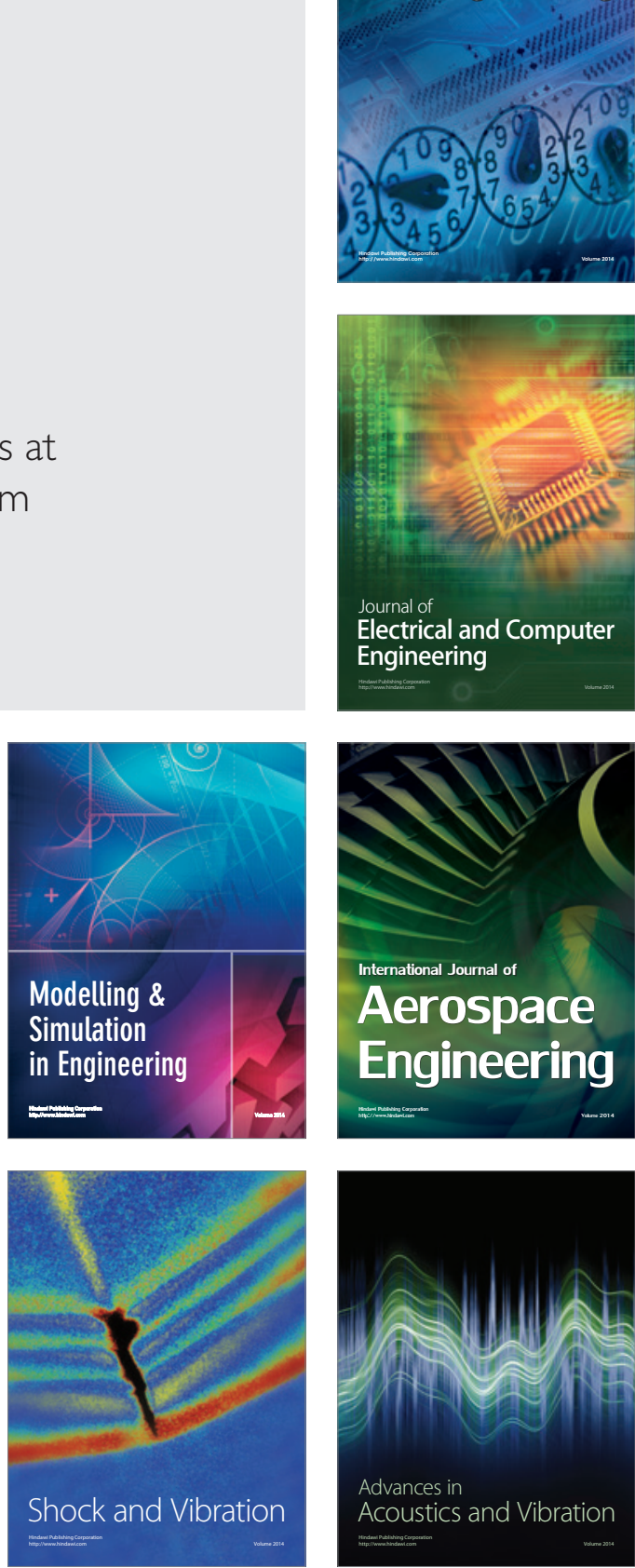